person-years of observation (pyo) for at least two of the three HIVgroups, i.e. a HIV-1 mono and/or a HIV-2 mono and/or a HIV-D infected group. Meta-analyses were performed using random effects models, estimating combined mortality rate ratios (MRRs).

Results Of the 631 identified titles, 7 articles met the inclusion criteria. Of these, 6 articles were included in the meta-analysis of HIV-D versus HIV mono-infected and 7 were included in the analysis of HIV-1 versus HIV-2 mono-infected people. The overall MRR of HIV-D versus HIV-1 was 1.11 (95\% CI 0.95-1.30). The overall MRR of HIV-D versus HIV-2 was 1.81 (95\% CI 1.43-2.30) and the MRR of HIV-1 versus HIV-2 was 1.86 (95\% CI 1.44-2.39)

Conclusion The mortality rate of HIV-D infected individuals did not significantly differ from the mortality rate of HIV-1 monoinfected individuals. The mortality rates of both HIV-1 monoinfected and HIV-D infected individuals were significantly higher than the rate among HIV-2 mono-infected individuals. Therefore, we conclude that HIV-2 mono-infected have a lower mortality rate than HIV-1 infected individuals, but there is no evidence that HIV-D infected individuals survive longer than HIV-1 monoinfected individuals.

\section{P3.204 HIV RISK BEHAVIOUR AMONG MEN WHO HAVE SEX WITH MEN IN YANGZHOU AND GUANGZHOU CHINA: A COHORT STUDY}

doi:10.1136/sextrans-2013-051184.0661

0 Wang, Y Yin, X Chen, G Liang, B Wang, R Zhang, N Jiang. Institute of Dermatology, Chinese Academy of Medical Sciences, Nanjing, China

Background This study provides the HIV prevalence, HIV incidence, HIV risk-factors, and demographics of Yangzhou MSM and a comparison of HIV risk behaviour among Yangzhou and Guangzhou MSM in China.

Methods A cross-sectional study and a prospective cohort study were conducted among MSM in Yangzhou and Guangzhou from July 2009 to September 2010. A total of 617 MSM (317 Guangzhou, 305Yangzhou) were screened for eligibility.

Results Compared with Guangzhou sample, Yangzhou sample was significantly older and lower educated and less likely to identify themselves as homosexual $(p<0.001)$, but more likely to be married $(p<0.001)$, and more likely to sex intercourse with female $(p<0.001)$. No significant differences were found in receiving more than 5 sexual health services in two samples $(p>0.05)$ and the proportions never or not always using condoms during sex with female and always use condoms in such a case $(p>0.05)$. A higher prevalence of HIV and syphilis was found among Yangzhou sample than Guangzhou sample ( $p<0.001)$. Aged (older than 40 years), married, and syphilis-positive were associated with HIV infection in both samples In Multivariate logistic regression analysis.

Conclusions There were significantly differences in demographic characteristics and risk sexual behaviours and the prevalence and incidence of HIV and syphilis between Yangzhou sample and Guangzhou sample. Our results showed that Yangzhou sample was at higher risk of infection of HIV than Guangzhou sample and reinforced the strong need for more and further investigation targeting MSM in economic-medium cities to prevent HIV in China.

\section{P3.205 DECLINING HIV PREVALENCE IN ZAMBIA: SENTINEL SURVEILLANCE PROGRAMMATIC INSIGHT AND THE NEED FOR HIV INCIDENCE DATA}

doi:10.1136/sextrans-2013-051184.0662

'S Kamocha, ${ }^{2} \mathrm{C}$ Mulenga, ${ }^{2} \mathrm{D}$ Mwakazanga, ${ }^{3} \mathrm{M}$ Monze, ${ }^{2} \mathrm{~N}$ Nyoni, ${ }^{1} \mathrm{~F}$ Soud, ' $\mathrm{M}$ Shields, 'M Marx. 'Centers for Disease Control and Prevention, Lusaka, Zambia; ${ }^{2}$ Tropical diseases research centre, Ndola, Zambia; '3niversity teaching hospital, Lusaka, Zambia
Background Antenatal clinic (ANC) HIV sentinel surveillance has provided key data to inform health policy, especially between population-based surveys. HIV prevalence among teenage attendees has been used as a proxy for incidence in Zambia, where incidence data are lacking. We present trends in HIV prevalence among ANC attendees in Zambia since 1994

Methods We assessed HIV prevalence in 15-39 year-old women accessing ANC in convenience sample-based cross-sectional surveys in 21 sites in 1994, 1998, 2002, 2004, 2006, 2008 and 2011. Anonymous blood was tested from consecutively-enrolled women on their first ANC visit; age, residence, parity and gravidity were also noted. We evaluated trends in HIV prevalence.

Results Among 8,222 women surveyed in 2011, HIV prevalence remained essentially unchanged from 2008 (16.2\% vs. $16.4 \%$, $p=0.74)$, but declined from a peak of $19.6 \%$ in1994 ( $p<0.001)$. Similarly, HIV prevalence stagnated among 1,661 15-19 year-olds between 2011 and 2008 (7.9\% vs. 8.3\% p = 0.66) but declined from its peak of $13.9 \%$ in 1994 ( $p<0.001)$. Declines in HIV prevalence among rural residents have been modest (11.8\% peak in 2004 vs. $10.4 \%$ in 2011 [ $n=2904$ ], $\mathrm{p}=0.09$ ). HIV prevalence among 5,318 urban women declined from $22.8 \%$ in 2008 to $19.6 \%$ in 2011 $(p<0.001)$, the lowest level since a peak of $27.6 \%$ in $1994(p<0.001)$. Among 904 15-19 year-old urban women HIV prevalence declined in $2011(10.1 \%$ ) from a peak of $16.4 \%$ in 2002 ( $p<0.001)$, but was essentially unchanged compared to $2008(11.1 \%, p=0.42)$.

Conclusions HIV prevalence declined in urban ANC attendees from 2008-2011 and in all surveyed ANC attendees compared to 1994. Results suggest success in prevention activities over the past 17 years, especially among urban women. Declines in prevalence have slowed recently, likely reflecting increased treatment-related survival possibly combined with decreases in incidence. Incidence and survival data are needed to fully understand these data.

\section{P3.206 CHARACTERIZING HIV SERO-DISCORDANCY AMONG STABLE COUPLES IN CAMBODIA, THE DOMINICAN REPUBLIC, HAITI, AND INDIA}

doi:10.1136/sextrans-2013-051184.0663

${ }^{1}$ H Chemaitelly, 1,2,3 $\mathrm{L}$ J Abu-Raddad. 'Infectious Disease Epidemiology Group, Weill Cornell Medical College-Oatar, Doha, Oatar; '2Department of Public Health, Weill Cornell Medical College, Cornell University, New York, NY, United States; ${ }^{3}$ Vaccine and Infectious Disease Institute, Fred Hutchinson Cancer Research Center, Seattle, WA, United States

Background Characterizing the epidemiology of HIV sero-discordancy among stable couples (SCs) is essential to inform HIV policy and programming, especially considering the recent availability of efficacious interventions among stable HIV sero-discordant couples (SDCs). This work complements a quantitative assessment of HIV sero-discordancy among SCs in sub-Saharan Africa (SSA), by analysing sero-discordancy in all countries outside SSA for which HIV biomarker demographic and health surveys (DHS) are available.

Methods We derived measures of HIV sero-discordancy using nationally-representative DHS data for Cambodia, the Dominican Republic (DR) including a sub-population at higher risk of HIV (Bateyes-DR), Haiti, and India. Vietnam was excluded from our analysis because of the low number of couples affected by HIV (3 couples).

Results HIV was more prevalent in Bateyes-DR (3.3\%) and Haiti $(2.2 \%)$ than in other settings $(<1 \%)$. About two-third of the population in reproductive age in these countries were engaged in SCs. The proportions of SCs affected by HIV and of SDCs were, respectively, $5.7 \%$ and $4.2 \%$ (Bateyes-DR), $4.7 \%$ and $3.2 \%$ (Haiti), $1.2 \%$ and $0.9 \%$ (DR), $1.0 \%$ and $0.5 \%$ (Cambodia), and $0.5 \%$ and $0.4 \%$ (India). Among SCs affected by HIV, 74.3\% were sero-discordant in BateyesDR compared to $68.3 \%$ in Haiti, $70.1 \%$ in DR, $51.6 \%$ in Cambodia, and $78.5 \%$ in India. About a third of HIV sero-positive persons had 